\title{
Monitoring Tissue Evolution on Electrodes with Bio-Impedance Test
}

\author{
A. Maldonado, P. Pérez, G. Huertas, A. Yúfera, A. Rueda, and J. L. Huertas \\ Instituto de Microelectrónica de Sevilla, CSIC, Sevilla. Spain \\ Universidad de Sevilla. Spain. \\ Email:\{maldonado, pablo.perez, gloria, yufera, rueda, huertas\}@imse-cnm.csic.es
}

\begin{abstract}
A technique for real-time monitoring of bioimpedances using a Voltage Oscillation (VO) methodology is proposed. The main idea relies on connecting the bio-system in such a way that a suitable electrical oscillator, which only uses a DC power source, is built. Thanks to the employed electrical models, the oscillation parameters can be directly related to the biological test. System simulations show that the impedance values of a tissue, called herein $Z_{x}$, can be determined by measuring the actual frequency and amplitude of the proposed VO system, being possible to select the frequency range to optimize the system sensitivity.
\end{abstract}

Keywords - Bioimpedance, tissue impedance, microelectrode model, oscillation-based systems.

\section{INTRODUCTION}

Today, many Lab-on-a-Chip (LoC) and implanted devices for biomedical applications are based on sensing the impedance of a biological sample as one of the main biomarkers [1]. The utility of this approach was demonstrated by the electrical impedance frequency-dependent response of biological materials, which reflects actual physical properties of a tissue, like conductivity and permittivity; in special, the $\beta$-dispersion ranging from kilohertz to hundreds of megahertz [2]. This relationship is mainly due to the cell shapes, cell membrane structures, and the amount of intra and extra cellular solution. Impedance-based techniques allow the use of non-invasive and relatively cheap equipment to extract relevant information from bio-samples, such as in cancer cell assays [3,4]; because of healthy tissues and cancer cells are different in shape, size and orientation. Glucose concentration in blood [5], tissue impedance evolution [6], cell-growth rate and toxicity [7, 8], bacterial detection [9], and others assays [10], are examples of several techniques that can benefit from this bio-marker. Furthermore, real-time monitoring or tissue supervision can benefit from bioimpedance markers to evaluate the degradation level of implanted devices for bodies hydration test [19], like ECG [20], neonatal function monitoring [21], Cardiac Resintonization Therapy (CRT) [22], blood Pulse Wave Velocity [23], liver tissue [24], neural recording devices [17], etc. Among other Impedance Spectroscopy (IS) techniques, Electrical Cell-substrate Impedance Spectroscopy (ECIS) was described [11] as a useful alternative for Cell Culture assays, allowing real-time monitoring, and greatly reducing the economic cost and human effort in those assays. Recently, an Oscillation-Based (OB) technique was proposed [12] enabling the extraction of information about cell growth in a cell culture, by measuring the oscillation parameters.

In this paper, we will apply the ideas behind the circuit configuration in [12] to study the state and time evolution of a tissue bio-impedance. To do that, several tasks must be completed; first of all, the electrical model of the system, the electrode-tissue under study, has to be properly defined [17]. Secondly, the OB circuits in [12] have to be tweaked for this particular application, to match both the working frequency range and the amplitude of interest; and finally, the system sensitivity range to the oscillation parameters must be studied and analysed. It is proposed herein to obtain the parametric curves describing the time evolution of the tissue impedance from measurements of the frequency and the amplitude of the proposed circuit oscillator.

Up today, most impedance measurement approaches consider the "excitation" at a given frequency and measure the obtained response [13, 14]. Alternatively, the VO approach converts the bio-system under test into an oscillator, adding some extra external components to force the "biological circuit" to oscillate, thus avoiding the use of any circuitry for both ac stimuli generation and accurate impedance measurements. To apply $\mathrm{VO}$ to $Z_{\mathrm{x}}$ measurements, a positive feedback loop is implemented. It is particularly important an accurate prediction of the oscillation parameters (frequency and amplitude), either analytically or by means of simulations [15], as well as avoiding a dependence of these 
parameters on any active element saturation levels. The solution proposes a non-linear element (comparator) within the feedback loop to guarantee self-maintaining oscillations [16], and a Band-Pass (BP) filter in the loop, as shown in Fig.1. There exists an oscillation condition, which has to be satisfied. As a consequence, the main oscillation parameters are function of the tissue impedance, $Z_{X}$.

In what follows, we will first analyze in section II the electrical model proposed for the micro-electrode-tissue system and the setup employed for measuring. The system blocks will be described and mathematically analyzed in section III, demonstrating the correct values for frequency and amplitude of oscillations obtained for the proposed test. Simulation results will be described in section IV, and finally, some conclusions will be summarized in section $\mathrm{V}$.

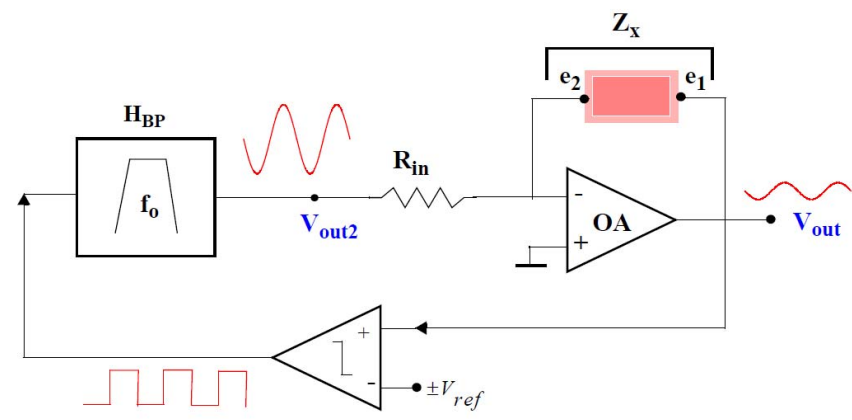

Fig. 1: Proposed VO system block diagram. A non-linear element, the comparator, and a $2^{\text {nd }}$-order Band-Pass filter, $H_{B P}$, with a central frequency, $f_{o}=8 \mathrm{kHz}$, are placed within the positive feedback loop to force oscillations.

\section{Electrode-Tissue ElectricAl Model}

How relevant is the Electrode-Tissue Electrical Model (ETEM) for testing bio-samples is an issue directly related to the setup employed [18]. In this paper, it is proposed to measure the tissue impedance (brain cortex [17]) with a bipolar, or also called, two-wires setup. In this case, the electrodes are on the signal path, and the voltage response measurement includes the tissue resistance plus the electrode response. Since the knowledge of ETEM is required for an adequate data extraction, we have considered the model proposed in [17] for each electrode, in a two-wires setup (Fig. $2)$. In that Figure, $R_{c t}$ is the transfer resistance, $C_{C P E}$ the constant phase impedance, $\mathrm{R}_{\mathrm{s}}$ the spreading resistance from each electrode through the medium up to the working electrode, and $Z_{x}$ includes the spreading resistance plus the tissue resistance under test. Values employed in the proposed analysis correspond to such those given in [17]. Therefore, if $\mathrm{Z}_{\mathrm{CPE}}=\left(2 \mathrm{C}_{\mathrm{CPE}} \cdot \mathrm{s}^{\beta}\right)^{-1}$, with $\beta=0.9$ then,

$$
H(s)_{\text {Tissue }+ \text { Electrode }}=\frac{2 R_{C T}+Z_{x}\left(1+s^{\beta} C_{C P E} R_{C T}\right)}{1+s^{\beta} C_{C P E} R_{C T}}
$$

being: $Z_{x}=R_{\text {encap }}+R_{\text {bulk }}\left(R_{\text {encap }}\right.$ changes, increasing with time, whereas $R_{\text {bulk }}$ is a constant value) $\mathrm{C}_{\mathrm{CPE}}=1.09 \mathrm{nF}, \mathrm{R}_{\text {bulk }}=20 \mathrm{k} \Omega$, $\mathrm{R}_{\mathrm{ct}}=3.85 \mathrm{M} \Omega, \mathrm{R}_{\text {encap }}=0 \Omega ; 200 \mathrm{k} \Omega ; 1 \mathrm{M} \Omega$. In Fig. 3 , it is displayed the Bode diagram of the tissue\&electrode impedance for three different values of $Z_{x} \quad\left(R_{\text {encap }}=0 \mathrm{k} \Omega\right.$, $200 \mathrm{k} \Omega$ and $1000 \mathrm{k} \Omega$ ) and for $\beta=0.9$ and $\beta=1$. Indeed, for a frequency of $8 \mathrm{kHz}$ (peak frequency for the considered BP filter), there exists an important change in the magnitude and the phase of the achieved Bode graphics.

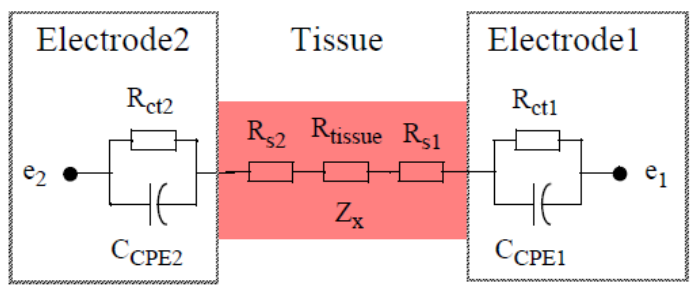

Fig. 2: Elements for the electrode-tissue electrical model: transfer resistance, $\mathrm{R}_{\mathrm{ct}}=3.85 \mathrm{M} \Omega$; Constant phase element, $\mathrm{C}_{\mathrm{CPE}}=1.09 \mathrm{nF}, \beta=0,9$ and $\mathrm{Z}_{\mathrm{x}}$ impedance, due to spreading resistance $\left(\mathrm{R}_{\mathrm{s}}\right)$ from each electrode through the saline solution or medium, and the tissue contribution. $Z_{\mathrm{x}}=20 \mathrm{k} \Omega$, initially for the case under study).
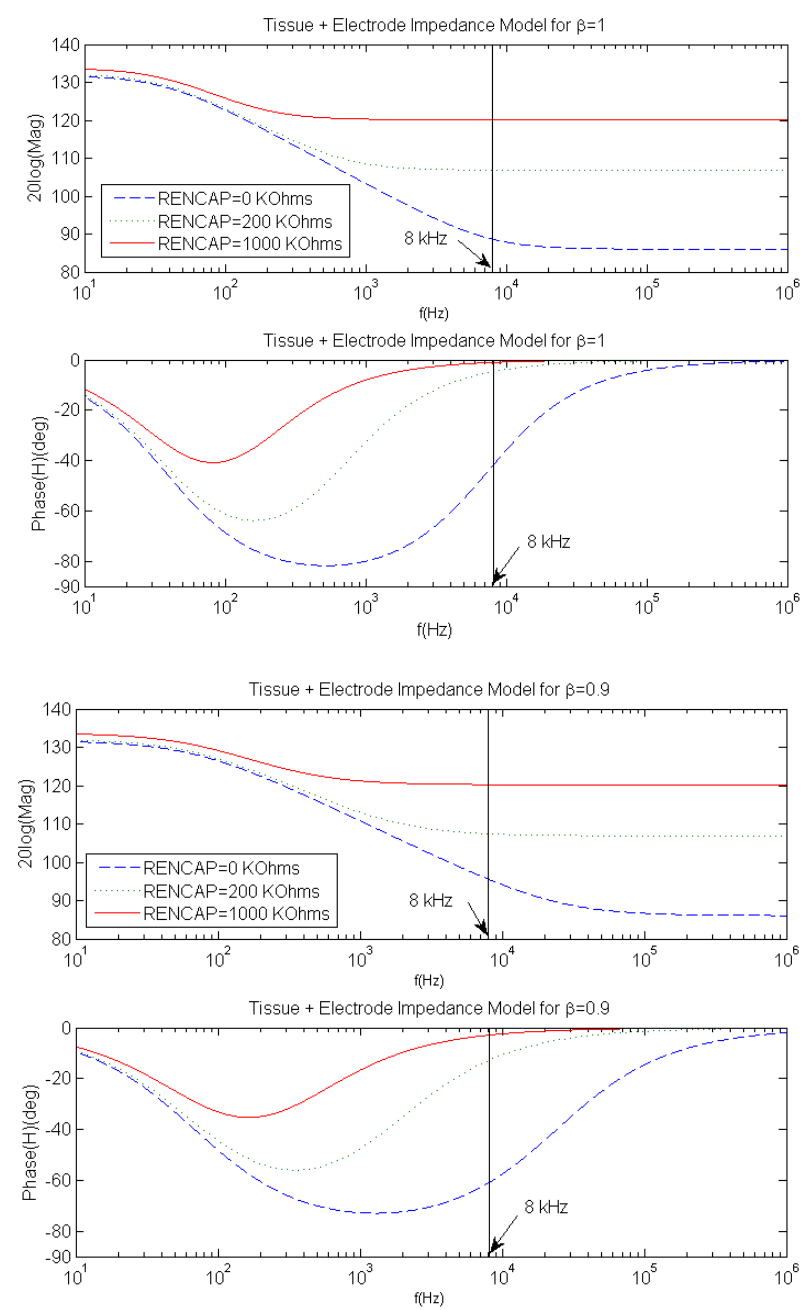

Fig. 3: Bode diagrams showing the magnitude and phase responses of the electrode-tissue model in [17], for, $\mathrm{Zx}$, of $20 \mathrm{k} \Omega, 220 \mathrm{k} \Omega$ and $1020 \mathrm{k} \Omega$. 
From curves in Fig. 3, and extracting the appropriate information from both cases $(\beta=0.9$ and $\beta=1)$, different values of magnitudes and phases in the working frequency for each particular $\mathrm{R}_{\text {encap }}$ are achieved (Table $\mathrm{I}$ ). Moreover, this result is even more significant for $\beta=0.9$.

Table I: Magnitude and phase of $\mathrm{H}(\mathrm{s})_{\text {tissue-electrode }}(8 \mathrm{kHz})$.

\begin{tabular}{|c|c|c|c|c|}
\hline \multicolumn{3}{|c|}{ Magnitude } & \multicolumn{2}{c|}{ Phase } \\
\hline RENCAP & $\beta=0.9$ & $\beta=1.0$ & $\beta=0.9$ & $\beta=1.0$ \\
\hline $\mathbf{0} \mathbf{\Omega}$ & $95.63 \mathrm{~dB}$ & $88.67 \mathrm{~dB}$ & $-61.19^{\circ}$ & $-42.26^{\circ}$ \\
$\mathbf{2 0 0} \mathbf{~} \boldsymbol{\Omega}$ & $107.40 \mathrm{~dB}$ & $106.90 \mathrm{~dB}$ & $-13.02^{\circ}$ & $-4.74^{\circ}$ \\
$\mathbf{1} \mathbf{M} \boldsymbol{\Omega}$ & $120.30 \mathrm{~dB}$ & $120.20 \mathrm{~dB}$ & $-2.95^{\circ}$ & $-1.03^{\circ}$ \\
\hline
\end{tabular}

\section{VO IMPLEMENTATION}

Mathematically, the characteristic equation of the system in Fig. 1 can be described as:

$$
1+N(a) H(s)=0
$$

where, $\mathrm{N}(\mathrm{a})=2 \mathrm{~V}_{\text {ref }} / \pi \mathrm{a}$ is the comparator describing-function $[15,16]$ and $\mathrm{H}(\mathrm{s})$ is the transfer function of the feedback loop.

The general BP transfer function will be given by,

$$
H_{B P}(s)=\frac{k_{1}^{*} \frac{\omega_{o}^{*}}{Q^{*}} s}{s^{2}+\frac{\omega_{o}^{*}}{Q^{*}} s+\omega_{o}^{* 2}}
$$

being $\omega_{0}{ }^{*}, Q^{*}$ and $k_{1}{ }^{*}$ the BP filter parameters. The corresponding tissue+electrode system transfer function is

$$
H(s)_{\text {Tissue+Electrode }}=\frac{2 R_{C T}+Z_{x}\left(1+s^{\beta} C_{C P E} R_{C T}\right)}{1+s^{\beta} C_{C P E} R_{C T}}
$$

where, as said above, the parameters $\left(\mathrm{R}_{\mathrm{CT}}, Z_{x}\right.$ and $\left.C_{C P E}\right)$ are directly related to the electrode size, technology and biological tissue. Then, the global linear function expression from Fig. 1 will be given by,

$$
H(s)=-H_{B P}(s) H_{\text {Tissue }+ \text { Electrode }}(s) / R_{\text {in }}
$$

To force the oscillations, a pair of complex poles of the overall system has to be placed on the imaginary axe. The way to determine the oscillation conditions (gain, frequency and amplitude) is solving equation (2). This is equivalent to find the solution of this equation:

$$
1+N(a) H(s)=\left(s^{2}+\omega_{O S C}^{2}\right)\left(s^{\beta}+A\right)=0
$$

if we consider $\beta=1$ (from the above expression we can suppose that the $\beta$ parameter only affects to the single pole), a first evaluation of the accomplished oscillations can be estimated, being the coefficients given by,

$$
\begin{aligned}
& A=\frac{R_{i n}+R_{i n} \frac{\omega_{o}^{*}}{Q^{*}} C_{C P E} R_{C T}-N\left(a_{o s c}\right) k_{1}^{*} \frac{\omega_{o}^{*}}{Q^{*}} Z_{X} C_{C P E} R_{C T}}{R_{i n} C_{C P E} R_{C T}} \\
& \omega_{o}^{2}=\frac{R_{i n} \cdot \frac{\omega_{o}^{*}}{Q^{*}}+\omega_{o}^{* 2} R_{i n} C_{C P E} R_{C T}-N\left(a_{o s c}\right) k_{1}^{*} \frac{\omega_{o}^{*}}{Q^{*}}\left(R_{C T}+Z_{X}\right)}{R_{i n} C_{C P E} R_{C T}} \\
& \omega_{o s c}^{2} \cdot A=\frac{R_{i n} \omega_{o}^{* 2}}{R_{i n} C_{C P E} R_{C T}}
\end{aligned}
$$

There exists an oscillatory solution and, without loss of generality, we can affirm that the actual oscillation result (as it can be seen in Fig. 3) will be closed to this first approximation. Therefore, these main oscillation parameters are function of tissue\&electrode impedance $\left(Z_{x}\right)$ as it will be shown in the next section for the frequency and amplitude of the oscillations.

The quality factor of the $\mathrm{BP}$ filter, $\mathrm{Q}^{*}$, regulates the closed-loop system oscillatory behaviour in Fig. 1. We need a trade-off between a well-defined oscillation output and enough margin in the oscillation frequency dynamic range. In this case, we selected $\mathrm{Q}=10$, because a clear resonance peak is attained and, conversely, a suitable slope in the phase curve is also obtained which produces a significant change in the oscillation frequency for the three values of $Z_{x}: 20 \mathrm{k} \Omega, 220$ $\mathrm{k} \Omega$ and $1020 \mathrm{k} \Omega$.

\section{IV.SIMULATION RESULTS}

The expected oscillation parameters, $f_{\text {osc }}$ and $A_{\text {osc }}$, are function of $Z_{x}$, as it is represented in Fig. 4 for a platinum electrode [17]. These curves were obtained using matlab simulations. The $f_{\text {osc }}$ increases monotonically in the range [7, $7.9] \mathrm{kHz}$, while the $A_{\text {osc }}$, increases, in the range of [0.03, 0.7] $\mathrm{V}$, when tissue impedance $Z_{\mathrm{x}}$ increases from $20 \mathrm{k} \Omega$ to 1020 $\mathrm{k} \Omega$. Due to the small voltage-level required at $V_{\text {out }}$, it can be considered the secondary output, $V_{\text {out } 2}$, as signal voltage output, improving the dynamic range. The obtained oscillation frequencies and amplitudes are shown in Fig. 4, where it can be observed a correlation with the tissue increments: high values of tissue impedance $Z_{x}$, give large oscillation amplitudes and lower oscillation frequencies, demonstrating the sensitivity of the proposed system to evaluate the actual tissue impedance status. Fig. 5 (c) displays the FFT analysis for the output signal $\mathrm{V}_{\text {out2 }}$ for the three studied values of $Z_{x}$. On the other hand, in Fig. 6 it is depicted how the $\mathrm{Q}$ factor changes the frequency oscillation range for the $Z_{x}$ range of interest. For our measurements, we have selected $\mathrm{Q}=10$. 

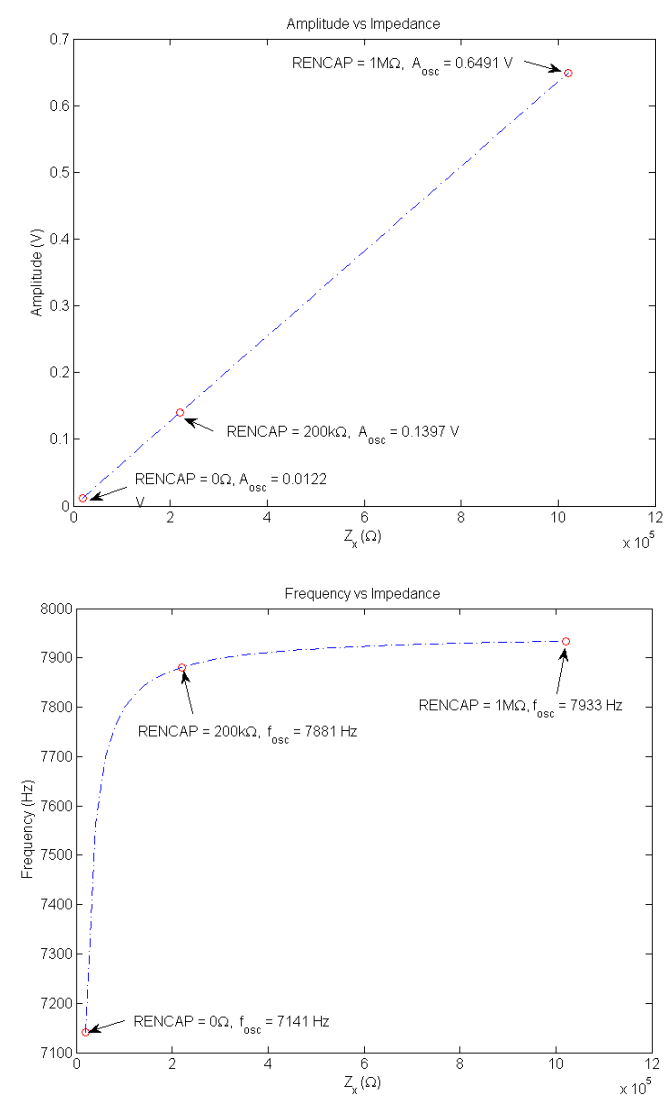

Fig. 4: Oscillation parameter obtained with matlab simulations for $Z_{\mathrm{x}}$ tissue of $20 \mathrm{k} \Omega, 220 \mathrm{k} \Omega$ and $1020 \mathrm{k} \Omega$, with $\beta=1$ : (a) Amplitude ( $\left.A_{\text {osc }}\right)$ and (b) frequency: $f_{\text {osc }}$, both measures at $V_{\text {out } 2}$ at circuit in Fig. 1.
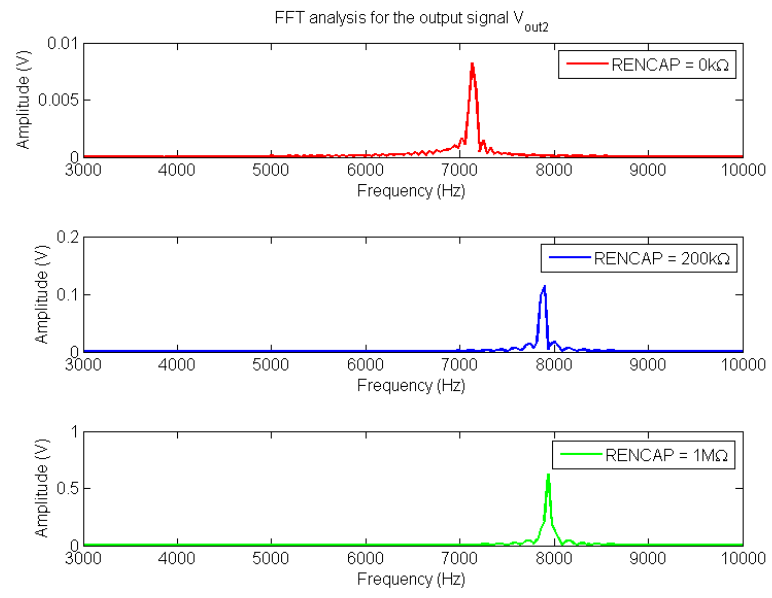

Fig. 5: FFT analysis of the output signals, $V_{\text {out }}$, at circuit in Fig. 1 obtained from matlab/simulink simulations (for $Z_{\mathrm{x}}$ tissue of $20 \mathrm{k} \Omega, 220 \mathrm{k} \Omega$ and 1020 $\mathrm{k} \Omega)$.

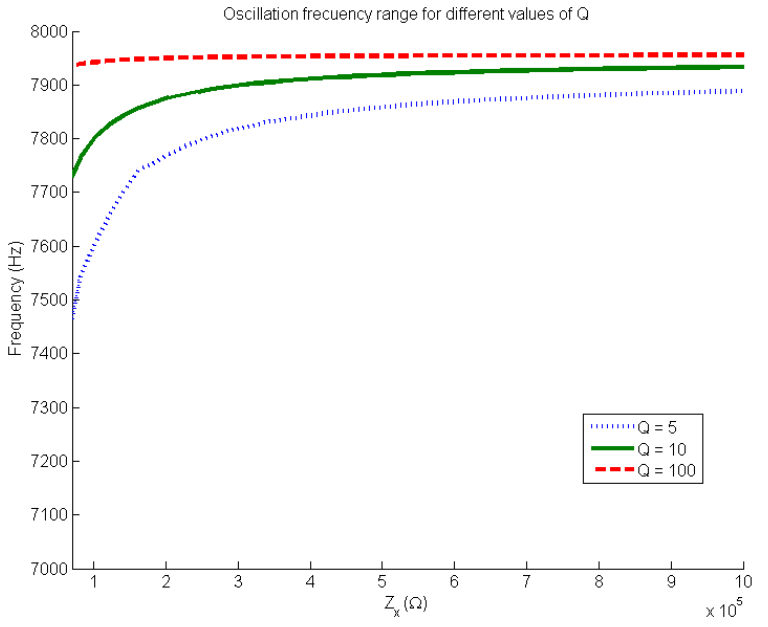

Fig. 6: Oscillation frequency: $f_{\text {osc }}$, versus $Z_{\mathrm{x}}$, for several $\mathrm{Q}$ values. It is observed how frequency dynamic range increases with lower quality factors.

\section{CONCLUSIONS}

This paper presents an approach to do bio-impedance measurements based on an oscillation-based method using oscillation parameters as sensing variables. Frequency and amplitude of achieved oscillations are function of the tissue impedance under test, enabling its real time monitoring to supervise its status. We have applied the scheme to the rat brain cortex electrical model in [17] and the obtained results showed how bioimpedance changes up to $1 \mathrm{M} \Omega$ can be detected, also increasing the sensor sensitivity for low bioimpedance values.

\section{ACKNOWLEDGMENT}

This work was supported in part by the Spanish founded Project: TEC 2013-46242-C3-1-P: Integrated Microsystem for Cell Culture Assays, co-financed with FEDER.

\section{REFERENCES}

[1] S. Grimnes, S. and O. Martinsen, "Bio-impedance and Bioelectricity Basics," Second edition. Academic Press, Elsevier. 2008.

[2] H. P. Schwan, "Electrical properties of tissue and cell suspensions," Advances in Biological and Medical Physics. New York, Academic press, vol. 5, pp. 147-224. 1957.

[3] I. Giaever \& C. R. Keese, "Micromotion of mammalian cells measured electrically, Proc. Nail. Acad. Sci. USA. Cell Biology, vol. 88, pp: 7896-7900. 1991.

[4] B. Blady \& B. Baldetorp, "Impedance spectra of tumour tissue in comparison with normal tissue; a possible clinical application for electrical impedance tomography. Physiol. Meas., vol. 17, suppl. 4A, pp. A105-A115. 1996.

[5] R. D. Beach et al., "Towards a Miniature In Vivo Telemetry Monitoring System Dynamically Configurable as a Potentiostat or Galvanostat for Two- and Three- Electrode Biosensors," IEEE TIM54, no 1, pp: 61-72. 2005.

[6] A. Yúfera et al., "A Tissue Impedance Measurement Chip for Myocardial Ischemia Detection," IEEE TCASI-52, no 12 pp: 26202628. 2005. 
[7] X. Huang et al., "Simulation of Microelectrode Impedance Changes Due to Cell Growth," IEEE Sensors J., 4, nº 5, pp: 576-583. 2004.

[8] P. Daza, A. Olmo, D. Cañete and A. Yúfera, "Monitoring Living Cell Assays with Bio-Impedance Sensors," Sensors and Actuators B, 176, pp: 605-610. 2013.

[9] S. Radke et al., "Design and Fabrication of a Microimpedance Biosensor for Bacterial Detection," IEEE Sensor J., vol 4, $\mathrm{n}^{\circ} 4,434$ 440. 2004.

[10] D. A. Borkholder. "Cell-Based Biosensors Using Microelectrodes," PhD Thesis, Stanford University. 1998.

[11] Giaever et al., "Use of Electric Fields to Monitor the Dynamical Aspect of Cell Behaviour in Tissue Culture," IEEE BME-33, $\mathrm{n}^{\circ} 2$, 1986.

[12] G. Huertas, A. Maldonado, A. Yúfera, A. Rueda, J. L. Huertas, "The Bio-Oscillator: A Circuit for Cell-Culture Assays," IEEE TCAS-II, IEEE. vol. 62, $\mathrm{n}^{\circ}$ 2, pp: 164-168, 2015.

[13] A. Manickam, et al., "A CMOS Electrochemical Impedance Spectroscopy (EIS) Biosensor Array," IEEE BIOCAS-4, n6. pp: 379390. 2010.

[14] A. Yúfera and A. Rueda, "Design of a CMOS closed-loop system with applications to bio-impedance measurements," MJ 41, pp: 231-239. 2010.

[15] G. Huertas et al., "Oscillation-Based Test in Mixed-Signal Circuits," (Frontiers in Electronic Testing). Springer. 2006.

[16] P. Fleischer et al, "A Switched Capacitor Oscillator with Precision Amplitude Control and Guaranteed Start-Up," JSSC-20, ${ }^{\circ}$ 2, 1985.

[17] A. Mercanzini, P. Colin, J. Bensadoun, A. Bertsch and P. Renaud, "InVivo Electrical Impedance Spectroscopy of Tissue Reaction to Microelectrode Arrays," IEEE TBE, vol. 56, n 7,pp:1909-1918,. 2009.

[18] Sohmyung Ha, Chul Kim,Yu M. Chi, Abraham Akinin, Christoph Maier, Akinori Ueno, and Gert Cauwenberghs, "Integrated Circuits and Electrode Interfaces for Noninvasive Physiological Monitoring," IEEE T-BioCAS, vol. 61, n. 5, pp: 1522-1537, 2014.

[19] B. Eilebrecht, J. Willkomm, A. Pohl, T. Wartzek, and S. Leonhardt, "Impedance Measurement System for Determination of Capacitive Electrode Coupling," TBioCAS, vol. 7, no. 5, pp: 682-689. 2013.

[20] B. Taji, S. Shirmohammadi, V. Groza, and I. Batkin, "Impact of SkinElectrode Interface on Electrocardiogram Measurements Using Conductive Textile Electrodes," IEEE TIM, vol. 63, nº. 6, pp: 14121422. 2014.

[21] P. Kassanos, L. Constantinou, I. F. Triantis, and A. Demosthenous, "An Integrated Analog Readout for Multi-Frequency Bioimpedance Measurements," IEEE Sensor J., vol 14, nº 8, pp: 2792-2800.. 2014.

[22] L. Yan, J. Pettine, S. Mitra, S. Kim, D. Jee, H. Kim, M. Osawa, Y. Harada, K. Tamiya, C. Van Hoof, and R. F. Yazicioglu, "A 13 Analog Signal Processing IC for Accurate Recognition of Multiple IntraCardiac Signals," TBioCAS, vol. 7, nº. 6, pp: 785-795. 2013

[23] W. Lee, and S. Cho, "Integrated All Electrical Pulse Wave Velocity and Respiration Sensors Using Bio-Impedance," IEEE JSSC, vol. 50, $\mathrm{n}^{\circ}$. 3, pp: 776-785. 2015.

[24] W. H. Huang, C. K. Chui, S. H. Teoh, and S. K. Y. Chang, "A Multiscale Model for Bioimpedance Dispersion of Liver Tissue, "IEEE TBE vol. 59, nº. 6, pp: 1593-1597. 2012. 